\title{
Avaliação de sondas de TDR multi-haste segmentadas para estimativa da umidade do solo ${ }^{1}$
}

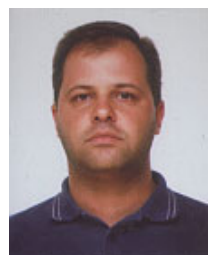

Claudinei F. Souza² \& Edson E. Matsura ${ }^{3}$

\author{
1 Parte da Tese de Doutorado do primeiro autor. Trabalho financiado pela FAPESP - Proc. 98/04931-7 e 13819-6 \\ 2 FEAGRI/UNICAMP. CP 6011, CEP 13081-970, Campinas, SP. E-mail: cfsouza@agr.unicamp.br (Foto) \\ ${ }^{3}$ FEAGRI/UNICAMP
}

Protocolo $180-27 / 12 / 2001$

\begin{abstract}
Resumo: O conhecimento da umidade do solo é de fundamental importância para a agricultura, sobretudo na determinação de sua variação, na otimização do manejo do solo e da água. Para medidas de umidade do solo existe tendência de utilização da técnica de TDR (Reflectometria no Domínio do Tempo) que, de certa forma, é nova no Brasil. Colaborando com a difusão da técnica, estudou-se a possibilidade de utilização de sondas multi-haste segmentadas em um equipamento de TDR. O trabalho foi dividido em duas partes, em que na primeira, as performances de duas configurações de sonda foram analisadas e, na segunda parte, as sondas foram avaliadas durante o monitoramento da umidade em condições de laboratório e de campo. Foi possível verificar-se a viabilidade do uso de sondas multi-haste segmentadas na estimativa da umidade de um perfil de solo; entretanto, a utilização da Sonda 1, com segmentação de $0,10 \mathrm{~m}$, ficou limitada à profundidade de $0,40 \mathrm{~m}$, devido à dificuldade da interpretação dos picos de impedância em sua extremidade.
\end{abstract}

Palavras-chave: reflectometria no domínio do tempo, guia de onda, manejo da água

\section{Multi-wire TDR probe evaluation to monitor soil water content}

\begin{abstract}
The knowledge of soil water content is important for agriculture, mainly the determination of its changes in the soil profile in order to optimize irrigation management. The tendency to use the technique of TDR (Time Domain Reflectometry) to measure the soil water content is relatively new in Brazil. To help the diffusion of this technique, the objective of this work was to study the possibility of using multi-wire probes with electrical impedance discontinuities in TDR equipment. The experiment was divided in to two parts. In the first one, the laboratory performance of two multi-wire probe configurations was studied; and in the second one, the evaluation of the probe reliability to monitor water content changes in porous media. The viability of the use of multiwire probes to estimate the water content in the porous media profile was confirmed. However, the use of a probe with $0.10 \mathrm{~m}$ segmentation was limited to the soil depth of $0.40 \mathrm{~m}$, due to the difficulty to interpret the impedance peaks at the extremity.
\end{abstract}

Key words: time domain reflectometry, wave guide, water management

\section{INTRODUÇÃO}

A água é extremamente importante na produção vegetal e o seu manejo racional é decisivo para o desenvolvimento das plantas, evitando a falta ou excesso. Desta maneira, o conhecimento da distribuição do teor de água (umidade) no solo torna-se cada vez mais necessário, uma vez que está intimamente ligado às propriedades do sistema solo-água-planta, onde o domínio desse conhecimento é, certamente, um dos fatores indispensáveis para uma agricultura sustentável e sem prejuízos ao meio ambiente.

Diversos são os métodos para a determinação da umidade do solo, como o gravimétrico, considerado o método direto padrão, além dos métodos indiretos, que estimam, a partir de outras propriedades do solo, tais como termalização de nêutrons, resistência elétrica e reflectometria no domínio do tempo (TDR).

O método gravimétrico apresenta resultados precisos a um custo relativamente baixo, quando usado como técnica laboratorial, mas para medidas no campo o acondicionamento e transporte das amostras podem reduzir a precisão dos resultados e, ainda, para amostragens repetidas em uma pequena área, podem causar interferência no local em estudo. O uso da sonda de nêutrons exige um treinamento especial para seu manuseio, principalmente pela presença de fontes radioativas. O bloco de gesso é um instrumento simples e de baixo custo, mas o elemento sensor sofre deterioração com o tempo devido à interação com os compostos físico-químicos do solo (Selker et al., 1993; Coelho \& Or, 1996). 
Para medidas de umidade do solo em laboratório e em campo, a utilização da TDR possui algumas vantagens quanto ao uso, principalmente pela precisão, possibilidade de multiplicação de leituras, repetição sem destruição da amostra de solo e pela segurança (Topp et al., 1980; Coelho \& Or, 1996). A TDR baseia-se no efeito da umidade do solo sobre a velocidade de propagação de pulsos de microondas em cabos condutores envoltos por solo. Este fenômeno se deve à diferença entre as constantes dielétricas, (K [adimensional]) da água, do ar e do material sólido do solo. $\mathrm{Na}$ matriz do solo as constantes dielétricas variam entre 1 e 81 . O ar possui o valor mínimo igual a 1 , as partículas sólidas variam entre 3 e 5, e a água possui o valor máximo 81 (Noborio, 2001). A constante $\mathrm{K}$ medida neste sistema (ar-solo-água) é denominada constante dielétrica aparente (Ka [adimensional]); assim, conhecendo-se esta variável, pode-se estimar a umidade volumétrica do solo por meio de calibrações obtidas a partir de dados de campo ou de laboratório (Conciani et al., 1996). Para a medição são necessários, basicamente, um testador de cabo e um sensor com hastes metálicas (sonda) ligados por um cabo coaxial.

O uso da TDR para estimar a umidade do solo tem sido limitado pelo custo, tanto do testador de cabo como da sonda, na qual existe ainda a limitação de uso em apenas uma profundidade, pois em geral as sondas são constituídas de hastes contínuas de aço inoxidável. Este fato tem sido contornado com a disponibilização de hastes segmentadas que, por diferença no diâmetro e material, permitem variação na impedância ao longo das mesmas e leituras em diferentes profundidades no perfil do solo (Topp \& Davis, 1985, Conciani et al., 1996 e Souza et al., 1999). Sabe-se, porém, que sua utilização não está difundida entre os usuários da técnica, devido à carência de informações descrevendo suas potencialidades e limitações, principalmente sobre a qualidade e a precisão de leituras versus configuração de sondas.

$\mathrm{O}$ desafio de se conhecer e utilizar essas informações, de forma correta, pode resultar em configurações de sondas com vantagens adicionais como, por exemplo, a possibilidade de leituras pontuais de umidade, tornando a técnica de TDR ainda mais atrativa como ferramenta capaz de auxiliar em estudos que envolvam a dinâmica da água no solo.

Este trabalho teve por objetivo desenvolver e avaliar sondas multi-haste segmentadas para se estimar a umidade do solo em condições de laboratório e de campo, utilizando-se um TDR-Trase System I*.

\section{MATERIAL E MÉTODOS}

\section{Performance das sondas multi-haste segmentadas}

A sonda multi-haste segmentada foi projetada para estimar a umidade do solo e suas dimensões são baseadas em uma linha de transmissão coaxial.

O uso desta sonda para estimar a umidade do solo não é novo (Topp \& Davis, 1985; Conciani et al., 1996; Souza et al., 1999) e algumas adaptações e mudanças se fizeram necessárias para melhorar o desempenho da mesma como, por exemplo, no sistema de fixação das hastes existentes no cabeçote da sonda, que aumentam a reflexão do sinal eletromagnético; adição de

Referências à marca registrada não constituem endosso por parte dos autores um segmento pontiagudo para facilitar a penetração da sonda no solo, e redução do comprimento dos segmentos visando obter estimativas pontuais de umidade.

As sondas multi-haste segmentadas desenvolvidas, consistem de um sensor com hastes metálicas com trechos de distintos valores de impedância. Esses trechos são facilmente obtidos pela variação dos diâmetros das hastes, interpretados utilizando-se um gráfico semelhante ao da Figura 1, a qual corresponde a uma sonda com cinco segmentos. As sondas multi-haste segmentadas em estudo foram fabricadas em latão, com exceção dos trechos intermediários de diâmetro menor, de aço inoxidável. Com o auxílio das barras de tempo do equipamento TDR-Trase, pode-se obter o tempo de deslocamento do sinal eletromagnético na sonda $\left(\Delta t=t_{n+1}-t_{n}\right)$. Na Figura 1, observa-se que $t_{1}$ é tempo no início da sonda (logo após o final do cabo coaxial) e $t_{5}$ é o tempo no final útil da sonda e, ainda, que os picos coincidem com o centro do trecho intermediário de diâmetro menor. Uma limitação pode ser visualizada nesta mesma figura, referente aos quinto e último segmentos, que não apresentam seu término evidenciado, prejudicando a determinação da umidade, motivo pelo qual a leitura do último segmento foi desprezada neste trabalho.

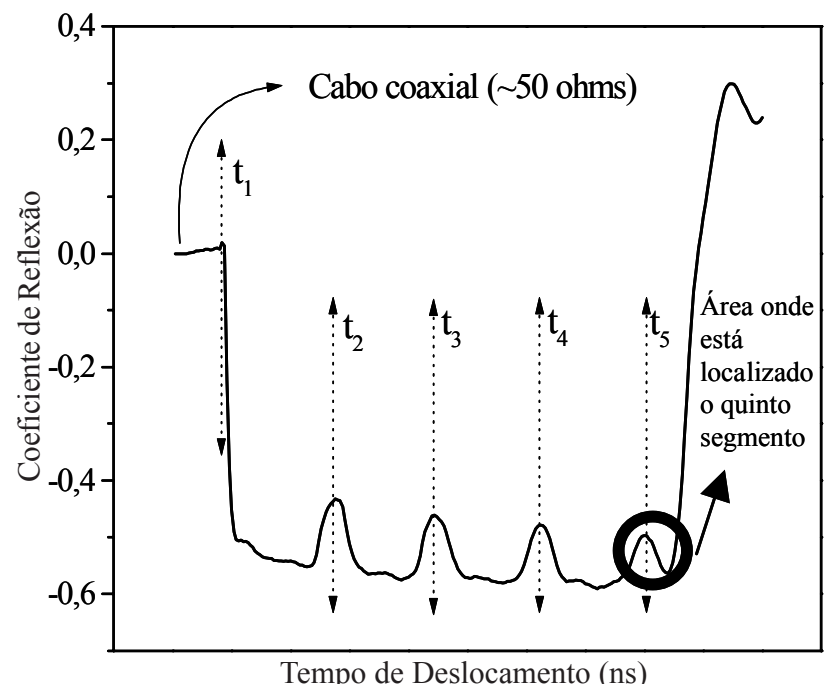

Figura 1. Esquema de interpretação da onda eletromagnética produzida por uma sonda multi-haste segmentada

$\mathrm{O} \Delta \mathrm{t}$ do sinal eletromagnético na sonda foi convertido para $\mathrm{Ka}$, por meio da equação abaixo, nos diferentes trechos (Soil Moisture, 1990).

$$
\mathrm{Ka}=\left(\frac{\Delta \mathrm{t} c}{\mathrm{~L}}\right)^{2}
$$

donde:

$$
\begin{array}{ll}
\Delta \mathrm{t} & \text { - tempo de deslocamento, } \mathrm{ns} \\
\mathrm{c} & \text { - velocidade da luz, } 0,30 \mathrm{~m} \mathrm{~ns}^{-1} \\
\mathrm{~L} & \text { - comprimento da haste, } \mathrm{m}
\end{array}
$$

As duas sondas desenvolvidas possuem 4 hastes com $0,675 \mathrm{~m}$ de comprimento e segmentação de 0,10 e 0,20 m denominadas, respectivamente, Sonda 1 e Sonda 2. A Sonda 1 foi dividida em 
7 segmentos e a Sonda 2 em 4 segmentos. As hastes foram construídas com o último segmento pontiagudo de $75 \mathrm{~mm}$ de comprimento, promovendo um perfil útil para leitura de $0,60 \mathrm{~m}$. O detalhamento da construção da sonda e suas dimensões, são apresentados na Figura 2.
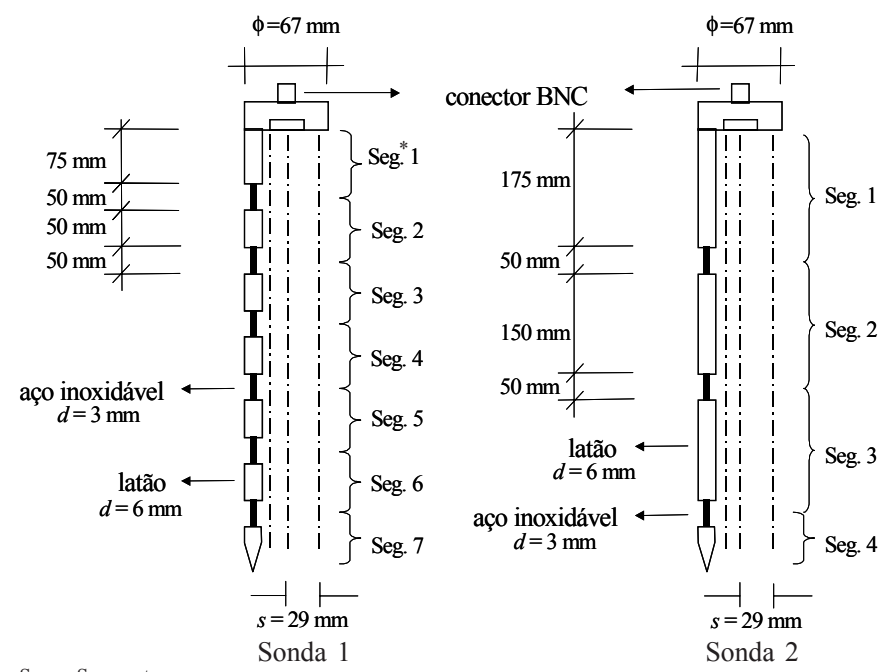

Seg. - Segmento

Figura 2. Esquema de segmentação das sondas 1 e 2

Avaliação do material construtivo da sonda: Conforme já descritas, as sondas desenvolvidas possuem parte de seus constituintes em latão, de menor custo de material e mão-deobra, em relação ao aço inoxidável, que tem sido o material dominante na construção de sondas nos últimos anos, sendo recentemente substituído pelo latão (Noborio, 2001).

Com o intuito de confirmar e se conhecer novas vantagens de como se usar hastes de latão em substituição ao aço inoxidável, realizou-se uma avaliação em laboratório, através de uma sonda do tipo "Wave Guide Connector", que possibilitou a troca das hastes contínuas (aço inoxidável/latão) para comparar leituras de Ka obtidas com as diferentes hastes que possuíam as mesmas dimensões, $6 \mathrm{~mm}$ de diâmetro e $150 \mathrm{~mm}$ de comprimento.

O solo utilizado foi um Latossolo Vermelho Distroférrico, coletado do campo experimental da Faculdade de Engenharia Agrícola/UNICAMP - SP, da camada de 0 - $0,20 \mathrm{~m}$; posteriormente, o solo foi destorroado, secado ao ar e peneirado (peneira de $2 \mathrm{~mm}$ ). As principais características físicas deste solo são apresentadas na Tabela 1.

Tabela 1. Características físicas do Latossolo utilizado

\begin{tabular}{rccccc}
\hline \multirow{2}{*}{$\begin{array}{c}\text { Camada } \\
(\mathrm{m})\end{array}$} & $\begin{array}{c}\text { Densidade } \\
\text { do Solo }\end{array}$ & $\begin{array}{c}\text { Porosidade } \\
\text { Total }\end{array}$ & \multicolumn{3}{c}{ Textura $\left(\mathrm{g} \mathrm{kg}^{-1}\right)$} \\
\cline { 5 - 7 } & $\left(\mathrm{kg} \mathrm{m}^{-3}\right)$ & $(\%)$ & Argila & Silte & Areia \\
\hline $0-0,25$ & 1240 & 53,20 & 560 & 90 & 350 \\
$0,25-0,50$ & 1230 & 53,70 & 610 & 80 & 310 \\
$0,50-0,80$ & 1210 & 54,30 & 650 & 70 & 280 \\
\hline
\end{tabular}

Para efetuar as determinações de Ka com as diferentes hastes, seguiu-se o procedimento descrito por Tommaselli (1997). Nesta metodologia, as etapas de umedecimento, com adição de $100 \mathrm{~mL}$ de água por etapa, foram continuamente repetidas, até se atingir umidades próximas à da saturação. $\mathrm{O}$ procedimento é assim explicado: metade da amostra de solo coletada, de cerca de $4 \mathrm{~L}$, foi esparramada em uma mesa, formando uma fina camada de solo e, através de um pulverizador, adicionou-se cerca de metade da água desejada $(50 \mathrm{~mL})$; a seguir, a outra metade da amostra foi esparramada sobre a metade anterior, pulverizando-se com água até completar os $100 \mathrm{~mL}$. O solo foi bem misturado e peneirado, de três a quatro vezes, em peneira de malha de $2 \mathrm{~mm}$, obtendo-se sua homogeneização. Após o umedecimento e homogeneização, o solo foi colocado em um recipiente plástico, de cerca de $5 \mathrm{~L}$ de volume, compactado de modo a manter a sua densidade o mais uniforme possível. O recipiente com o solo foi pesado, o que permitia a obtenção do valor de densidade do solo úmido, valor este a ser utilizado na determinação da umidade do solo.

Impedância característica das sondas multi-haste segmentadas: A impedância característica (Zo $[\Omega])$ é o modo de se expressar a lei que relaciona a variação de voltagem e corrente, com distância ao longo de uma linha de transmissão uniforme, geralmente utilizada para se avaliar a performance de sondas de TDR. Em geral, sondas com Zo superior a $200 \Omega$ são inadequadas para determinação da umidade do solo pois, neste caso, a reflexão prejudica a interpretação do sinal eletromagnético, inviabilizando a técnica. A Zo de uma linha coaxial de transmissão ideal depende exclusivamente da geometria da linha (Zegelin et al., 1989) e pode ser calculada por:

$$
\mathrm{Zo}=60 \ln \left(\frac{2 \mathrm{~s}}{\mathrm{~d}}\right)
$$

sendo:

s $\quad$ - raio do condutor externo, $\mathrm{m}$

d - diâmetro do condutor interno, $\mathrm{m}$

Segundo Zegelin et al. (1989) e Heimovaara (1993) pode-se medir Zo por determinação do coeficiente de reflexão da sonda (p) isto quando a mesma está envolta por um material dielétrico uniforme, através da expressão:

$$
\mathrm{Zo}=\mathrm{Zu} \mathrm{K}{ }^{0,5} \frac{(1+\mathrm{p})}{(1-\mathrm{p})}
$$

que:

$\mathrm{Zu} \quad$ - Impedância no cabo $\sim 50 \Omega$

$$
\mathrm{p}=\left(\frac{\mathrm{V}_{1}}{\mathrm{~V}_{0}}\right)-1
$$

Sendo $V_{0}$ a amplitude do sinal eletromagnético gerado pelo TDR, e $V_{1}$ a amplitude do sinal eletromagnético depois da reflexão do início da sonda.

Para as sondas em estudo, pôde-se estimar Zo usando-se a Eq. 2 através das dimensões de s e d e compará-lo com o medido na Eq. 3; além disso, adicionaram-se à avaliação, duas sondas comuns de TDR contínuas, respectivamente P1 e P2. As Sondas $\mathrm{P} 1$ e P2, comprimento $\mathrm{L}=0,10$ e $0,20 \mathrm{~m}$, respectivamente, foram adicionadas à avaliação, para oferecer critério e confiabilidade 
às discussões dos resultados, uma vez que são utilizadas em diversos experimentos, o que se levou a assumi-las como sondas ideais para estimativas de umidade do solo. O material dielétrico usado foi água destilada a $20^{\circ} \mathrm{C}(\mathrm{K}=81)$.

Estimativa da umidade em condições de laboratório e de campo Avaliação das sondas multi-haste segmentadas em laboratório: A avaliação foi realizada com uma areia classificada como grossa, um meio poroso homogêneo que permitiu isolar-se os possíveis efeitos oriundos do meio, avaliando-se o efeito das diferentes configurações das sondas.

A areia foi secada ao ar para se obter a umidade baixa e, após, acondicionada em duas colunas de PVC, $0,25 \mathrm{~m}$ de diâmetro e $0,9 \mathrm{~m}$ de comprimento, de tal forma a se obter uma densidade de $1650 \mathrm{~kg} \mathrm{~m}^{-3}$.

Após a instalação das sondas, aplicou-se $1 \mathrm{~L}$ de água sobre a superfície da areia, por meio de um emissor (tipo gotejador) com 4 saídas e taxa de aplicação de $8 \mathrm{~L} \mathrm{~h}^{-1}$. Essas saídas foram distribuídas uniformemente ao redor da sonda, obtendo-se uma boa homogeneidade do umedecimento da areia, de cima para baixo.

O processo dinâmico da água na areia foi monitorado durante $8 \mathrm{~h}$ através de leituras de $\mathrm{Ka}$ e comparados os resultados obtidos. Para esta comparação, calcularam-se médias entre as umidades estimadas nos segmentos 1 e 2,3 e 4 e 5 e 6 , para a Sonda 1, podendo-se verificar e comparar o comportamento das estimativas de umidade em profundidades semelhantes entre as Sondas 1 e 2, conforme Figura 2. Entendendo-se melhor o que foi realizado, teoricamente as sondas devem possuir o mesmo comportamento em relação ao posicionamento de seus picos, para uma condição dielétrica semelhante. Desta forma, os picos de impedância números 1, 2 e 3 da Sonda 2, devem ser coincidentes com os picos 2, 4 e 6, da Sonda 1 .

Finalmente, determinaram-se os correspondentes valores de umidade volumétrica com base na curva de calibração, descrita por Topp et al. (1980).

Avaliação da sonda multi-haste segmentadas em campo: Utilizou-se, neste ensaio, uma sonda semelhante à Sonda 2. Fez-se esta opção pelo fato dos resultados da avaliação em laboratório indicarem ser o uso desta confiável para se descrever a umidade do perfil do solo em profundidade superior a $0,40 \mathrm{~m}$, quando comparado com a Sonda 1. O solo utilizado nesta etapa foi o Latossolo Vermelho Distroférrico, com as mesmas características descritas anteriormente.

Irrigou-se uma área experimental de $2 \times 2$ m elevando-se o máximo possível a umidade do solo, que corresponde a um valor de $0,40 \mathrm{~m}^{3} \mathrm{~m}^{-3} \mathrm{e}$, em seguida, instalou-se a sonda. Este procedimento facilitou a instalação da sonda, pois a penetração das hastes seria dificultada pela dureza de solo argiloso seco, de tal forma que o paralelismo entre as hastes foi mantido, fator importante para a obtenção de um sinal eletromagnético com características de reflexão que viabilizem sua interpretação.

Durante o período de $10 \mathrm{~d}$ tomou-se leitura diária das sondas que, em seguida, foram convertidas em constante dielétrica aparente do solo, nos diferentes trechos.

\section{RESULTADOS E DISCUSSÃO}

\section{Performance das sondas multi-haste segmentadas}

Avaliação do material construtivo da sonda: Verificou-se coeficiente de correlação satisfatório $(r=0,99)$ entre as leituras de Ka obtidas com diferentes materiais (aço inoxidável versus latão) e coeficiente angular da equação de regressão próximo de 1 (Figura 3). Esses resultados tanto comprovam existir uma associação linear entre os materiais como indicam uma variação direta no valor de Ka do aço inoxidável, para uma unidade de Ka do latão, comprovando-se que suas características apresentam relação funcional na propagação de ondas eletromagnéticas, ao longo das hastes metálicas. Desta forma, hastes de latão podem substituir as de aço inoxidável, promovendo economia na construção de sondas de TDR, uma vez que o preço do latão é inferior à mesma quantidade de aço inoxidável e oferece maior facilidade operacional para serviços de usinagem.

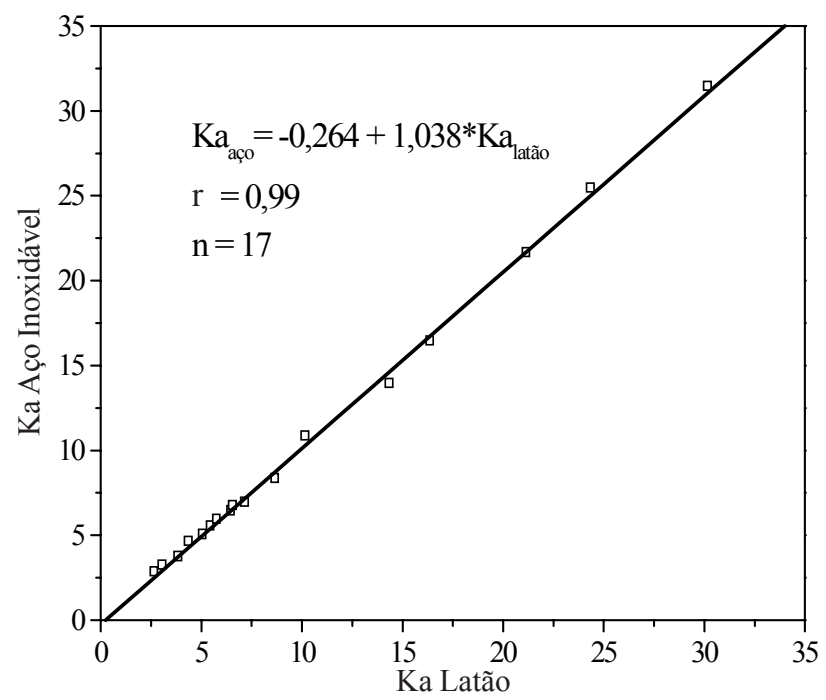

Figura 3. Correlação entre hastes de aço inoxidável e latão

Impedância característica das sondas multi-haste segmentadas: São apresentados, na Tabela 2, os resultados da impedância característica das sondas em estudo. Analisando-se os resultados

Tabela 2. Impedância característica das sondas multi-haste segmentada

\begin{tabular}{|c|c|c|c|}
\hline \multirow[b]{2}{*}{ Sondas } & \multirow{2}{*}{$\begin{array}{l}\text { Dimensões } \\
\mathrm{s} / \mathrm{d}, \mathrm{mm}\end{array}$} & \multicolumn{2}{|c|}{$\mathrm{Zo}[\Omega]$} \\
\hline & & $\begin{array}{l}\text { Geométrico } \\
\text { Eq. (1) }\end{array}$ & $\begin{array}{l}\text { Medido } \\
\text { Eq. (2) }\end{array}$ \\
\hline \multicolumn{4}{|c|}{ Sonda $1\left(\mathrm{n}^{\mathrm{o}}\right.$ de $\left.\mathrm{s}\right)$} \\
\hline 1 & $29 / 6$ & 136 & 171 \\
\hline 2 & $29 / 6$ & 136 & 170 \\
\hline 3 & $29 / 6$ & 136 & 169 \\
\hline 4 & $29 / 6$ & 136 & 168 \\
\hline 5 & $29 / 6$ & 136 & 167 \\
\hline 6 & $29 / 6$ & 136 & 166 \\
\hline \multicolumn{4}{|c|}{ Sonda $2\left(n^{\circ}\right.$ de $\left.s\right)$} \\
\hline 1 & $29 / 6$ & 136 & 168 \\
\hline 2 & $29 / 6$ & 136 & 167 \\
\hline 3 & $29 / 6$ & 136 & 166 \\
\hline Sonda P1 & $10 / 2,1$ & 135 & 198 \\
\hline Sonda P2 & $14 / 3,1$ & 132 & 196 \\
\hline
\end{tabular}


obtidos pela Eq. 2, observa-se que os valores calculados para as sondas multi-haste segmentadas são próximos aos valores das sondas comuns de TDR (P1 e P2). Assim, verificou-se, também, que as dimensões geométricas utilizadas são satisfatórias. Zegelin et al. (1989) apresentaram valores medidos de impedância que não ultrapassaram $173 \Omega$ para sondas multi-haste contínuas, com 4,76 mm de diâmetro e $150 \mathrm{~mm}$ de comprimento, valores estes considerados satisfatórios pelos autores.

Observa-se, na Figura 4 que, para a Sonda 1, pode-se encontrar os picos de impedância não muito bem definidos devido ao fenômeno de reflexão, dificultando a leitura. Para ambas as sondas, porém, é possível verificar que o coeficiente de reflexão diminui com o aumento do tempo de deslocamento, sendo mais evidente na Sonda 1 dentro da área circundada, cujo fenômeno é provocado pela interface entre materiais de diferentes propriedades elétricas. Utilizando-se a Eq. 2 verifica-se uma impedância igual a 136 e $177 \Omega$ para os segmentos em latão e aço inoxidável, respectivamente. Salienta-se que esta perda é causada pelo dimensionamento das hastes, que possuem diferentes materiais e geometria ao longo de seu comprimento; esta limitação dificulta a leitura para a determinação exata do pico de impedância causando, assim, erros na estimativa da umidade do solo. Segundo Heimovaara (1993) o problema deve agravar-se para solos secos e/ou salinos.

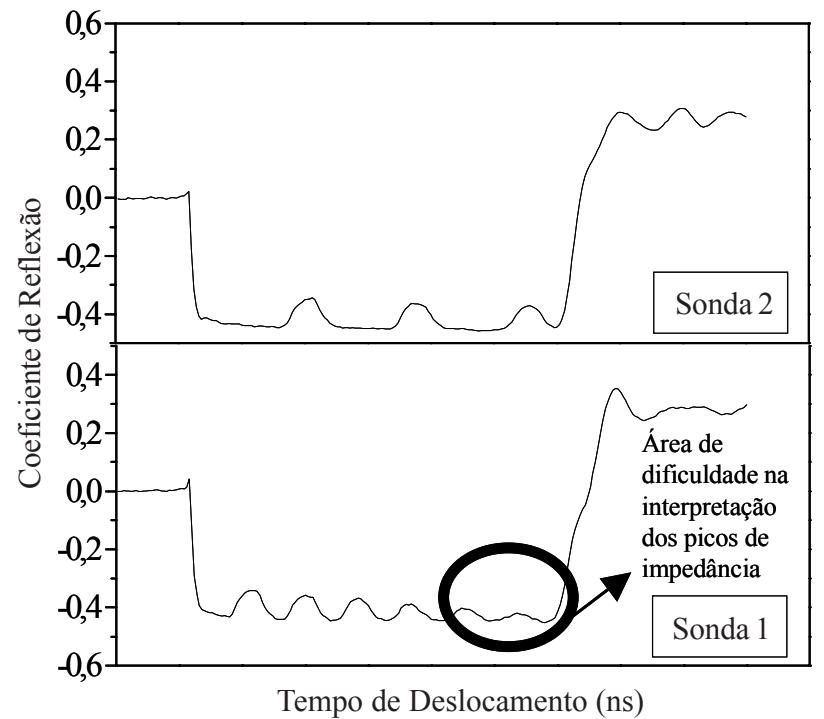

Figura 4. Comparação de leituras do tempo de deslocamento do sinal

Estimativa da umidade em condições de laboratório e de campo Avaliação das sondas multi-haste segmentadas em laboratório: Tem-se, na Tabela 3, uma comparação entre os principais resultados do monitoramento do processo de dinâmica da água em meio poroso, verificando-se que não há diferenças nas estimativas de umidade entre as diversas sondas, comprovando-se que ambas possuem características semelhantes na propagação de ondas eletromagnéticas ao longo da sonda; entretanto, observa-se também que, para a Sonda 1 e em sua extremidade, existe dificuldade na interpretação dos picos de impedância (Tabela 3); estes resultados confirmam as observações anteriores, mostrando que o número elevado de segmentações aumenta consideravelmente as reflexões do sinal. Em concordância com
Tabela 3. Comparação entre resultados da umidade volumétrica $(\theta)$ em função do tempo para as Sondas 1 e 2

\begin{tabular}{ccccccc} 
& \multicolumn{5}{c}{ Umidade Volumétrica, $\mathrm{m}^{3} \mathrm{~m}^{-3}$} \\
\cline { 2 - 7 } Tempo & $\begin{array}{c}\text { Camada } \\
(\mathrm{h})\end{array}$ & $(0-0,20 \mathrm{~m})$ & $\begin{array}{c}\text { Camada } \\
(0,20-0,40 \mathrm{~m})\end{array}$ & $\begin{array}{c}\text { Camada } \\
(0,40-0,60 \mathrm{~m})\end{array}$ \\
\cline { 2 - 7 } & \multicolumn{5}{c}{ Sonda } \\
\cline { 2 - 7 } & 1 & 2 & 1 & 2 & 1 & 2 \\
\hline $0: 00$ & 0,04 & 0,04 & 0,04 & 0,04 & $\mathrm{NE}^{*}$ & 0,04 \\
$0: 10$ & 0,14 & 0,13 & 0,04 & 0,04 & $\mathrm{NE}$ & 0,04 \\
$0: 20$ & 0,26 & 0,26 & 0,09 & 0,08 & $\mathrm{NE}$ & 0,04 \\
$0: 30$ & 0,21 & 0,21 & 0,17 & 0,17 & $\mathrm{NE}$ & 0,04 \\
$1: 15$ & 0,17 & 0,17 & 0,23 & 0,23 & $\mathrm{NE}$ & 0,04 \\
$3: 10$ & 0,14 & 0,14 & 0,20 & 0,20 & $\mathrm{NE}$ & 0,08 \\
$4: 00$ & 0,13 & 0,12 & 0,20 & 0,20 & $\mathrm{NE}$ & 0,10 \\
$5: 00$ & 0,13 & 0,12 & 0,18 & 0,18 & $\mathrm{NE}$ & 0,11 \\
$6: 10$ & 0,11 & 0,11 & 0,18 & 0,18 & $\mathrm{NE}$ & 0,12 \\
$8: 00$ & 0,11 & 0,11 & 0,18 & 0,18 & $\mathrm{NE}$ & 0,12 \\
\hline
\end{tabular}

"NE - Não estimado devido à dificuldade na interpretação dos picos de impedância

tais observações, Topp \& Davis (1985) apresentaram resultados que salientam esta dificuldade de interpretação dos gráficos, quando foram utilizadas sondas com mais de cinco segmentos.

Foi possível identificar-se variações de umidade durante o movimento dinâmico da água no perfil de $0-0,60 \mathrm{~m}$ da coluna de areia. Verificou-se, para ambas as sondas, que a maior umidade $\left(0,26 \mathrm{~m}^{3} \mathrm{~m}^{-3}\right)$ na profundidade de 0 - $0,20 \mathrm{~m}$ ocorreu aos $20 \mathrm{~min}$ a qual, após $1 \mathrm{~h}$, foi reduzida de forma rápida e, simultaneamente, monitorou-se o movimento da água para a próxima camada $(0,20-0,40 \mathrm{~m})$. Após $3 \mathrm{~h}$ do início do ensaio observou-se acréscimo de umidade na última camada $(0,40$ $0,60 \mathrm{~m}$ ) apenas para a Sonda 2, cujo resultado, porém, não descarta por completo a Sonda 1, sendo possível sua utilização em perfis de até $0,40 \mathrm{~m}$ de profundidade com detalhamento da umidade solo, ou seja, a cada $0,10 \mathrm{~m}$.

Avaliação da sonda multi-haste segmentada em campo: Observa-se, na Figura 5, a caracterização da umidade do perfil do solo em um processo dinâmico. Ocorreram sucessivas diminuições no armazenamento da água no solo e reposição de $8 \mathrm{~mm}$ por chuva entre os dias 5/9 e 6/9 (representada pela área

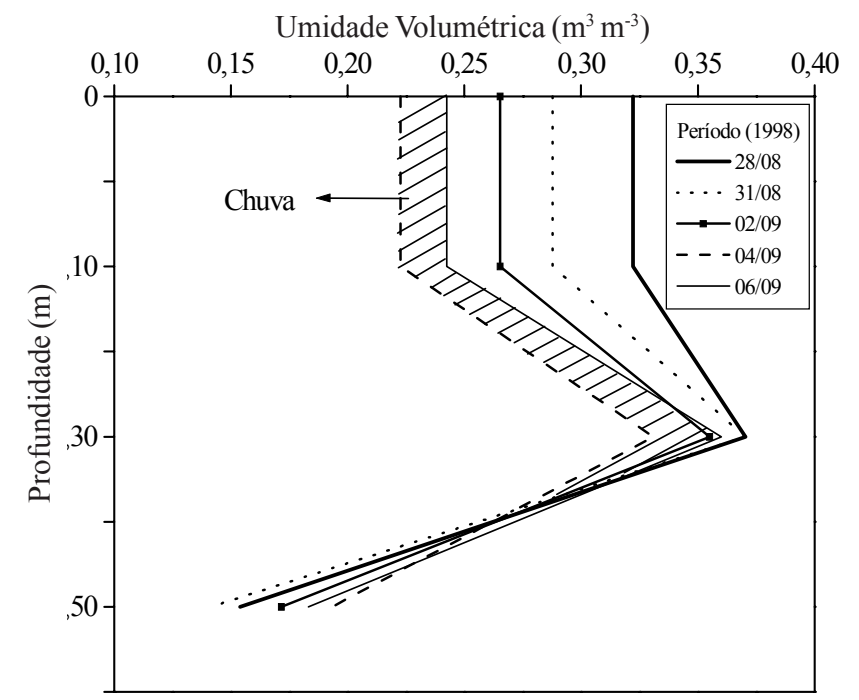

Figura 5. Variação da umidade no perfil do solo, ao longo do período estudado 
hachuriada); estas variações foram identificadas no período estudado, aplicando-se a regra do trapézio (Libardi, 2000) para o cálculo das variações do armazenamento.

Para trabalhos futuros, sugere-se avaliar novas configurações de sondas com segmentos menores, possibilitando um detalhamento ainda maior da umidade em perfis de solo.

\section{CONCLUSÕES}

1. A utilização de hastes de latão em substituição às de aço inoxidável, mostrou ser satisfatória, do ponto de vista técnicoeconômico.

2. As diferentes configurações das sondas utilizadas mostraram-se aptas para a determinação da umidade do solo em processos dinâmicos da água em laboratório e campo; entretanto, verificou-se limitação do uso de sondas segmentadas, de $0,10 \mathrm{~m}$.

3. Existe viabilidade no uso de sondas multi-haste segmentadas, na estimativa da umidade de um perfil de solo.

\section{AGRADECIMENTOS}

À Financiadora de Estudos e Projetos - FINEP, pelo financiamento que permitiu a aquisição do equipamento TDR; à Fundação de Amparo à Pesquisa do Estado de São Paulo FAPESP, pela concessão da bolsa de doutorado para o primeiro autor, e aos pesquisadores Dr. José Dalton Cruz Pessoa (CNPDIA/EMBRAPA) e Professor Dr. Tadeu José Garcia Tommaselli (FCT/UNESP) pelo apoio técnico.

\section{LITERATURA CITADA}

Coelho, E.F.; Or,D. Flow and uptake patterns affecting soil water sensor placement for drip irrigation management. Transactions of the ASAE, St. Joseph, v.39, p.2007-2016, 1996.

Conciani, W.; Herrmann, P.S.P.; Machado, S.L.; Soares, M.M. O uso da técnica de reflectometria no domínio do tempo (TDR) para determinação da umidade do solo in situ. Revista Solos e Rochas, São Paulo, v.19, p.189-199, 1996.
Heimovaara, T.J. Design of triple-wire time domain reflectometry probes in practice and theory. Soil Science Society of America Journal, Madison, v.57, p.1410-1417, 1993.

Libardi, P.L. Dinâmica da água no solo. 2. ed. Piracicaba: snt, 2000.509p.

Noborio, K. Measurement of soil water content and electrical conductivity by time domain reflectometry: A review. Computers and Electronics in Agriculture, Amsterdam, v.31, p.213-237, 2001.

Noborio, K.; Mclnnes, K.J.; Heilman, J.L. Measurements of cumulative infiltration and wetting front location by time domain reflectometry. Soil Science, Baltimor, v.161, p.480-483, 1996.

Selker, J.R.; Graff, L.; Steenhuis, T. Noninvasive time domain reflectometry moisture measurement probe. Soil Science Society of America Journal, Madison, v.57, p.934-936, 1993.

Soil Moisture Equipament Corporation. Trase System I. Santa Bárbara: Soil Moisture Equipament Corporation, 1990. 53p.

Souza, C.F.; Matsura, E.E.; Testezlaf,R. Desempenho de sondas multi-haste segmentada para o monitoramento da umidade do solo por meio da técnica de TDR. In: Congresso Brasileiro de Engenharia Agrícola, 28, 1999, Pelotas. Resumos... Pelotas: Sociedade Brasileira de Engenharia Agrícola, 1999. CDRom

Tommaselli, J.T.G. Influência de algumas características do solo sobre a calibração de um aparelho de TDR (Time Domain Reflectometry). Piracicaba: ESALQ-USP, 1997. 167p. Tese Doutorado

Topp, G.C.; Davis, J.L. Measurement of soil water content using time domain reflectometry (TDR): A field evaluation. Soil Science Society of America Journal, Madison, v.49, p.19-24, 1985.

Topp, G.C.; Davis, J.L.; Annan, A.P. Electromagnetic determination of soil water content: measurements in coaxial transmission lines. Water Resources Research, Washington, v.16,p.574-582, 1980.

Zegelin, S.J.; White, I.; Jenkins, D.R. Improved fields probes for soil water content and electrical conductivity measurements using TDR. Water Resources Research, Washington, v.25, p.2367-2376, 1989. 